\title{
Primer registro en el Pacífico suroriental de la anguila abisal Diastobranchus capensis Barnard, 1923 (Osteichthyes: Synaphobranchidae)
}

\author{
First record of the abyssal eel Diastobranchus capensis Barnard, 1923 in the \\ southeastern Pacific ocean (Osteichthyes: Synaphobranchidae) \\ Pablo Reyes ${ }^{1}$ y Germán Pequeño ${ }^{1}$ \\ ${ }^{1}$ Universidad Austral de Chile, Instituto de Zoología “Ernst F. Kilian” \\ Casilla 567, Valdivia, Chile \\ mardechile@gmail.com
}

\begin{abstract}
The finding of one specimen (IZUA-PM-2338, 875 mm TL) of Diastobranchus capensis Barnard, 1923 (Osteichthyes: Anguilliformes: Synaphobranchidae), captured at $2.000 \mathrm{~m}$ depth off Golfo de Penas, southern Chile (48 $31^{\prime} 12^{\prime \prime}$ 'S $-75^{\circ} 15^{\prime} 04^{\prime \prime} \mathrm{W}$ ) is reported and represents the first record of this genus for the southeastern Pacific. The
\end{abstract}

ichthyogeographical meaning of this finding is also analyzed. A taxonomic key to recognize Chilean synaphobranchid eels is included.

Key words: Continental slope, eel, abyss, Synaphobranchidae, Diastobranchus, Chile
La región denominada Pacifico suroriental constituye una de las zonas oceánicas más vastas del planeta. Dentro de esta área se encuentra el mar de Chile, una región que abarca más de cinco millones de kilómetros cuadrados de superficie marina, de las cuales hay muchas partes aún inexploradas, lo que ha derivado en que la ictiofauna de este sector diste mucho de estar bien conocida (Pequeño 2000). En los últimos veinte años ha existido un relevante avance en relación con la composición taxonómica de la ictiofauna marina de Chile, con una serie de aportes que, aisladamente, han contribuido a modificar los conceptos sistemáticos, zoogeográficos, ecológicos, pesqueros y otros, sobre los peces. El valor de los diferentes nuevos registros ha permitido estructurar el conocimiento científico actual de la ictiofauna chilena (Pequeño et al. 1992), compuesta por más de 1.100 especies (Pequeño 1989, 1997), a las cuales se siguen sumando especies a veces nuevas para la región, u otras veces nuevas para la ciencia, a medida que se desarrollan cruceros científicos en aguas de la Zona Económica Exclusiva (ZEE) y de acuerdo con el desarrollo de prospecciones de nuevos recursos, que conlleva la exploración de nuevos caladeros (Meléndez 1999). El problema de la composición taxonómica, distribución geográfica y origen de la ictiofauna de este sector suroriental del Pacífico, pese a los avances señalados, aún constituye un gran vacío y tan amplia zona parece guardar información fundamental para responder aquellos trascendentales problemas ictiológicos (Mead 1970).

En la presente investigación se adhiere al llamado realizado por Pequeño (1989) sobre la necesidad de informar los nuevos registros como forma de contribuir a mejorar el conocimiento de los peces de Chile y del Pacífico suroriental, presentando una adición a la ictiofauna chilena, constituida por Diastobranchus capensis Barnard, 1923(Synaphobranchidae: Anguilliformes).

La historia de la familia Synaphobranchidae en aguas chilenas es reciente, incorporándose a nuestra ictiofauna en el año 1991, con el registro de dos ejemplares de Synaphobranchus kaupi Johnson, 1862 capturados en el talud continental del norte de Chile por Kong \& Meléndez (1991). También se incorporó a la región a Synaphobranchus affinis Günther, 1877, sobre la base de registros enfrente de la costa norte de Chile (Sulak \& Shcherbachev 1997).

El ejemplar que motiva este estudio se obtuvo de un lance de prueba realizado con espineles de fondo en el golfo de Penas (48³1'12',S-75'15'04'’W) a una profundidad de $2.000 \mathrm{~m}$, por la lancha artesanal "Don Alfredo", dedicada a la extracción del bacalao de profundidad Dissostichus eleginoides Smitt, 1898, durante la temporada estival 2003. Se emplearon 
anzuelos Mustang $\mathrm{N}^{\circ} 5$ con carnada de anchoveta, Engraulis ringens Jenyns, 1842. La muestra fue fijada en formalina al $10 \%$, rotulada y almacenada en un contenedor plástico de $20 \mathrm{~L}$. Posteriormente fue remitida al Instituto de Zoología de la Universidad Austral de Chile para su estudio y conservación.

Las medidas del ejemplar fueron registradas con pié de metro de precisión de 0,1 mm. La definición de los caracteres observados, las mediciones, cuantificaciones y determinación taxonómica del ejemplar se realizaron siguiendo los trabajos de Castle (1986), Gon (1990) y Sulak \& Shcherbachev (1997).

Las abreviaturas en el texto corresponden a: $\mathrm{AB}$ longitud abertura branquial; AC ancho máximo de la cabeza; AM altura mayor corporal; AMC altura mayor de la cabeza; AMCO ancho mayor corporal; ANA altura abertura nasal anterior; ANP altura abertura nasal posterior; AO ancho oral; BA base de la aleta anal; BD base de la aleta dorsal; DNAH distancia narina anterior a extremo de hocico; DNPH distancia narina posterior a extremo de hocico; DO diámetro de la órbita; DON distancia ojo a abertura nasal posterior; IN ancho interorbital; IZUA-PM, es el acrónimo de la colección de peces marinos del Instituto de Zoología de la Universidad Austral de Chile; LC longitud de la cabeza; LE longitud estándar; LMS longitud mandíbula superior; LMI longitud mandíbula inferior; LNA longitud abertura nasal anterior; LP longitud de la aleta pectoral; LPR longitud pre pectoral; PA longitud preanal; PD longitud predorsal; PRO longitud preorbitaria. El estudio arrojó los siguientes resultados:

\section{Orden Anguilliformes}

Familia Synaphobranchidae

Diastobranchus capensis Barnard, 1923 (Fig. 1)

Material examinado: IZUA-PM 2338, un ejemplar de $875 \mathrm{~mm}$ LT.

\section{Breve descripción}

Cuerpo alargado, comprimido lateralmente. Mayor altura corporal presente a nivel del ano, siendo la cabeza de menor altura, al igual que la porción corporal posterior al ano, que se prolonga formando una cola larga que se adelgaza progresivamente hacia el extremo posterior y fuerte. La aleta anal es de base más larga que la dorsal. Ambas se originan en el tercio anterior del cuerpo y confluyen en la aleta caudal formando una sola aleta. Cuerpo con escamas pequeñas, ordenadas como tejido de canasto. Línea lateral sinuosa, se origina en la cabeza, cerca del sector superior del ojo, en proyección dorsal; finaliza en el ápice de la cola, con recorrido medio por el tronco y está provista de poros sensoriales. Ojos grandes y redondos. Mandíbula inferior más larga que la superior. Dientes presentes en ambas mandíbulas, fuertes, cortos, numerosos y con punta fina. Hocico largo, puntiagudo, se extiende sobre la proyección horizontal del ojo. Dos pares de narinas presentes. Las primarias adelante del ojo, frente a su sector medio. Secundarias conspicuas, tubulares, de posición subterminal en el hocico. Aleta pectoral con radios, el de mayor longitud es el más cercano al sector dorsal, los demás disminuyen progresivamente en longitud hacia el más próximo al sector ventral. Aleta pélvica ausente. Su color es burdeo oscuro, interrumpido por la conspicua línea lateral blanquecina.

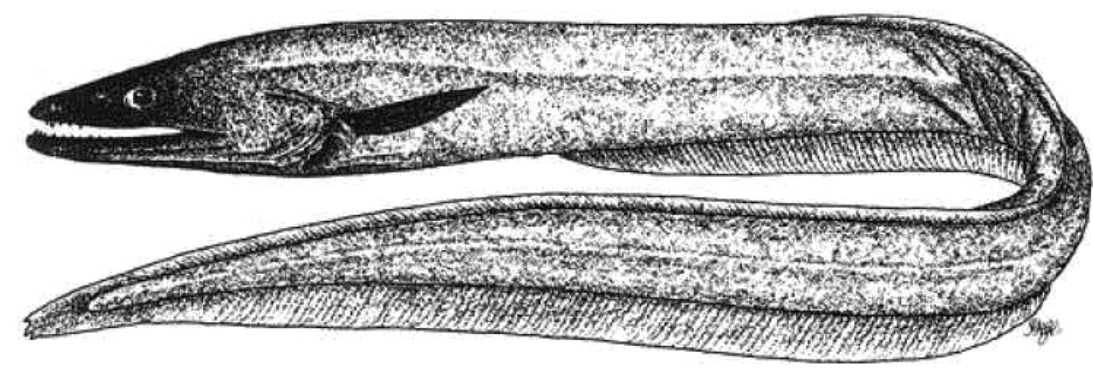

Figura 1

Diastobranchus capensis Barnard, 1923. Ejemplar de 112 cm (modificado de Castle 1986)

Diastobranchus capensis Barnard, 1923. Specimen 112 cm (modified from Castle 1986) 


\section{Morfometría y merística}

Los resultados de las mediciones efectuados en el ejemplar de este estudio, sobre la base de porcentajes en la LT y LC, se presentan en la Tabla 1. Hay muy pocos antecedentes de este tipo en la literatura, lo cual impide comparaciones que son deseables. En todo caso, un autor (Gon 1990) ha usado porcentajes sobre medidas decimales y ha proporcionado la oportunidad de algunas comparaciones que, en términos generales coinciden con nuestras observaciones (Tabla 1).

\section{Tabla 1}

Datos morfométricos de $D$. capensis (IZUA-PM 2338) expresados en porcentaje de la LT y porcentaje de la LC

Morphometric data of D. capensis (IZUA-PM-2338), given in percentage of the TL length and percentage of the HL

a) En porcentaje de la LT, comparados con datos de Gon (1990):

\begin{tabular}{ccc}
\hline Medida & $\begin{array}{c}\text { Presente estudio } \\
\text { \% LT }\end{array}$ & $\begin{array}{c}\text { Gon (1990) } \\
\text { \% LT }\end{array}$ \\
\hline LT & 100 & 100 \\
LE & 99,8 & - \\
LC & 11,4 & $12,1-14,6$ \\
LPR & 14,6 & - \\
LP & 9,6 & $6,6-6,8$ \\
PA & 21,6 & $21,4-24,9$ \\
PD & 35,9 & $29,5-37,5$ \\
AM & 9,3 & - \\
BD & 65,3 & - \\
BA & 58,3 & - \\
AMCO & 5,9 & - \\
\hline
\end{tabular}

b) En porcentaje de la LC, solamente el ejemplar objeto de este estudio:

\begin{tabular}{ccc}
\hline Medida & $\mathrm{mm}$ & $\% \mathrm{LC}$ \\
\hline LC & 140 & 100 \\
DO & 19,5 & 13,9 \\
IN & 22,7 & 16,2 \\
LMS & 82,5 & 58,9 \\
LMI & 80,5 & 57,5 \\
PRO & 41,2 & 29,4 \\
AO & 32 & 22,9 \\
AMC & 61,5 & 43,9 \\
AC & 39,5 & 28,2 \\
AB & 28 & 20 \\
LNA & 5,5 & 3,9 \\
ANA & 3,8 & 2,7 \\
DNAH & 13,2 & 9,4 \\
DNPH & 30,2 & 21,6 \\
ANP & 4,5 & 3,2 \\
DON & 5 & 3,57 \\
\hline
\end{tabular}

\section{Distribución geográfica}

Circumantártica. Pacífico suroccidental; Australia (Paxton et al. 1989) y Nueva Zelanda (Paulin et al. 1989). Atlántico suroccidental: Islas Malvinas (Brickle \& Laptikhovsky 2002) y Brasil (Menezes et al. 2003). Atlántico suroriental: Sudáfrica (Castle 1986). Océano Austral: Islas Kerguelen (Gon 1990). La actual comunicación provee su primer registro en el Pacífico suroriental y amplía su distribución geográfica a todos los extremos australes de los continentes del Hemisferio Sur. De paso, el presente registro es muy cercano al límite latitudinal sur conocido de la especie, al sur de Nueva Zelanda (Sulak \& Sccherbachev 1997).

\section{Distribución batimétrica}

De 180 a 1.400 m (Gon 1990); 183-1750 m (Sulak \& Shcherbachev 1997). La actual comunicación amplía su distribución batimétrica hasta los $2.000 \mathrm{~m}$ de profundidad.

La presencia de $D$. capensis en Sudamérica ha sido detectada recientemente en las islas Malvinas (Brickle \& Laptikhovsky 2002) y Brasil (Menezes et al. 2003), con esos aportes se extendió la distribución geográfica de la especie a casi todos los extremos australes de los continentes del Hemisferio Sur, lo que ha permitido señalar a este representante del Orden Anguilliformes como un pez de distribución gondwánica. Sin embargo, aún no había registros de la especie en el Pacífico suroriental. Por eso, este hallazgo tiene la importancia de dar mayor continuidad a la distribución geográfica de esta especie. Podría decirse que con este caso, sumado a muchos análogos, poco a poco, el Pacífico suroriental se va constituyendo en un área de insospechada diversidad ictiofaunística, lo cual concuerda parcialmente con las predicciones de Mead (1970), acerca del origen y composición se la fauna de peces del Océano Pacífico.

La amplia dispersión de la especie en el Hemisferio Sur puede estar relacionada con la masa de agua del Océano Austral, dentro de cuyos límites ha sido registrada (Gon 1990), o con las aguas profundas del Pacífico, por tratarse de una especie batidemersal (Castle 1986), e incluso puede vincularse con sistemas de montes sumergidos, como se ha observado en Nueva Zelanda, donde se la vincula con la pesquería del “orange roughy”, Hoplostethus atlanticus Collett, 1889, de la cual constituye fauna acompañante con aportes importantes de biomasa (McClatchie \& Coombs 2005). Sin embargo, tal situación no ha sido reportada en la 
pesquería del mismo recurso efectuada en los montes sumergidos de la ZEE de Chile (Instituto de Fomento Pesquero y Universidad Austral de Chile, 2003, Oñate 2005), por lo tanto queda en duda su calidad de acompañante de $H$. atlanticus en esa cordillera sumergida. Ante las evidencias, será oportuno consignar nuevos antecedentes de captura para la especie en aguas chilenas, que permitan estructurar un mayor conocimiento acerca de ella y de sus relaciones biogeográficas con áreas profundas del Hemisferio Sur. Por el momento se puede estimar con un alto grado de seguridad futuros reencuentros en Chile, ya que el ejemplar capturado se encuentra en edad reproductiva, debido a su longitud.

Hasta la fecha, el escaso conocimiento del talud continental profundo de la zona austral del Pacífico suroriental, especialmente al sur de la isla Mocha $\left(38^{\circ}\right.$ $30^{\prime} \mathrm{S}^{\circ}$ ) en Chile, indicaba la presencia de las familias Macrouridae, Zoarcidae, Chimaeridae y Squalidae, y de los ordenes Notacanthiformes y Ophidiiformes (Pequeño 2000). El ejemplar estudiado de Diastobranchus capensis proviene del golfo de Penas, Chile, a $2.000 \mathrm{~m}$ de profundidad y proporciona el primer registro para el Pacífico suroriental de la familia Synaphobranchidae, que pasa a integrar el conjunto de peces abismales patagónicos, junto con los taxa señalados.

De tal manera, dicha familia queda representada en el Pacífico suroriental por tres especies, que pueden reconocerse mediante la siguiente clave:

1 (2) Aberturas branquiales ventrolaterales, oblicuas .Diastobranchus capensis

2 (1) Aberturas branquiales ventrales, horizontales 3

3 (4) Aleta dorsal se inicia prácticamente en el mismo nivel que el inicio de la anal; 142 a 151 vértebras; longitud de la boca mayor que la distancia entre la boca y la base de las aletas pectorales

Synaphobranchus kaupi

4 (3) Aleta dorsal se inicia claramente por detrás de la inserción de la anal; 128 a 139 vértebras; longitud de la boca menor que la distancia entre la boca y la base de las aletas pectorales

\section{Agradecimientos}

A los Srs. Claudio Ferrada y Francisco Pinto, en nombre de la tripulación del buque pesquero "Don Alfredo", por obtener el material de estudio. A León Matamala (IZUA), por su apoyo en el laboratorio. Este estudio contó con el apoyo parcial del proyecto DID-S-200503, de la Universidad Austral de Chile al co-autor.

\section{Literatura citada}

Brickle P \& V Laptikhovsky. 2002. New records of deep-sea fishes from the waters around the Falkland Islands. Journal of Fish Biology 60(2): 492-494.

Castle P. 1986. Synaphobranchidae. En: Smith MM \& PC Heemstra (eds). Smiths' sea fishes, pp. 188-190. JLB Smith Institute of Ichthyology, Grahamstown. CTP Books Printers, Cape Town, Sudáfrica.

Gon O. 1990. Synaphobranchidae. En: Gon O \& PC Heemstra (eds). Fishes of the Southern Ocean, pp. 102-104. JLB Smith Institute of Ichthyology, Grahamstown, Sudáfrica.

Instituto de Fomento Pesquero \& Universidad Austral de Chile. 2003. Catálogo fauna íctica asociada a la pesquería chilena de orange roughy (Hoplostethus atlanticus). Subsecretaria de Pesca. Valparaíso, 52 pp.

Kong I \& $\mathbf{R}$ Meléndez. 1991. Estudio taxonómico y sistemático de la ictiofauna de aguas profundas capturada

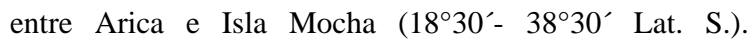
Estudios Oceanológicos 10: 1-81.

McClatchie S \& R Coombs. 2005. Low target strength fish in mixed species assemblages: the case of orange roughy. Fisheries Research 72: 185-192.

Mead GW 1970. A history of South Pacific fishes. En: Wooster W (ed). Scientific Exploration of the South Pacific, pp. 236-251. National Academy of Sciences, Washington D.C.

Meléndez R. 1999. Nuevas adiciones a la ictiofauna mesopelágica y demersal entre isla Mocha y la Península

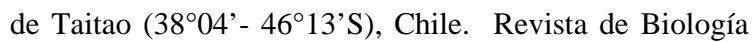
Marina y Oceanografía 34(2): 139-143.

Menezes N, A Buckup, L de Figueiredo \& L de Moura. (eds) 2003. Catálogo das espécies de peixes marinhos do Brasil. Museu de Zoologia de Universidade de São Paulo. Brasil, 160 pp. 
Oñate J. 2005. Ictiofauna del sector austral de la cordillera submarina de Nazca, frente al norte de Chile y sus relaciones biogeográficas. Tesis de Biología Marina, Universidad Austral de Chile, Valdivia, 77 pp.

Paulin C, A Stewart, C Roberts \& P McMillan. 1989. New Zealand fish: a complete guide. National Museum of New Zealand Miscellaneous Series 19, 279 pp.

Paxton J, D Hoese, G Allen \& J Hanley. 1989. Pisces. Petromyzontidae to Carangidae. Zoological Catalogue of Australia Vol. 7, 665 pp. Australian Government Publishing Service, Canberra.

Pequeño G. 1989. Peces de Chile, Lista Revisada y Comentada. Revista de Biología Marina, Valparaíso 24(2): 1-132.
Pequeño G. 1997. Peces de Chile, Lista Revisada y Comentada: Addendum. Revista de Biología Marina y Oceanografía, Valparaíso 32(2): 77-94.

Pequeño G. 2000. Delimitaciones y relaciones biogeográficas de los peces del Pacifico suroriental. Estudios Oceanológicos 19: 53-76.

Pequeño G, A Cea-Egaña \& W Sielfeld. 1992. Primer registro en Chile para tres especies de peces teleósteos marinos, en base a fotografías. Boletín Sociedad de Biología de Concepción, Chile 63: 169-173.

Sulak KJ \& YN Shcherbachev. 1997. Zoogeography and systematics of six living genera of Synaphobranchid eels, with a key to taxa and description of two new species. Bulletin of Marine Science 60 (3): 1158-1194.

Recibido el 28 de diciembre de 2005 y aceptado el 27 de septiembre de 2006 